\title{
Skin tests with SARS-CoV2 vaccine excipients in patients with first-dose mucous-cutaneous adverse reactions: a purpose of allergologic workup
}

Leonardo Bianchi ${ }^{1}$, Filippo Biondi ${ }^{1}$, Katharina Hansel ${ }^{1}$, Nicola Murgia ${ }^{2}$, Alessandro D'Arpino $^{3}$, Marta Tramontana ${ }^{1}$, and Luca Stingeni ${ }^{1}$

${ }^{1}$ Section of Dermatology Department of Medicine and Surgery University of Perugia Italy ${ }^{2}$ Section of Occupational Medicine Respiratory Diseases and Toxicology Department of Medicine and Surgery University of Perugia Italy

${ }^{3}$ Hospital Pharmacy Unit Santa Maria della Misericordia Hospital Azienda Ospedaliera di Perugia Italy

September 25, 2021

Article type: letter to the editor

Skin tests with SARS-CoV2 vaccine excipients in patients with first-dose mucous-cutaneous adverse reactions: a purpose of allergologic workup

Running title: skin tests with COVID-19 vaccine excipients

Authors: Leonardo Bianchi ${ }^{1}$, Filippo Biondi ${ }^{1}$, Katharina Hansel ${ }^{1}$, Nicola Murgia ${ }^{2}$, Alessandro D'Arpino ${ }^{3}$, Marta Tramontana ${ }^{1}$, Luca Stingeni ${ }^{1}$

1 Section of Dermatology, Department of Medicine and Surgery, University of Perugia, Italy

2 Section of Occupational Medicine, Respiratory Diseases and Toxicology, Department of Medicine and Surgery, University of Perugia, Italy

3 Hospital Pharmacy Unit, Santa Maria della Misericordia Hospital, Azienda Ospedaliera di Perugia, Italy

Corresponding author: Leonardo Bianchi, Section of Dermatology, Department of Medicine and Surgery, University of Perugia, Italy. E-mail: leonardo.bianchi@unipg.it

Keywords: SARS-CoV2 vaccine; excipients; adverse drug reaction; skin test; patch test; skin prick test; intradermal test

Word count: 629

Number of references: 6

Number of tables: 2

Number of figures: 0

Funding sources: None

Conflict of interest:

Dr. Bianchi has nothing to disclose 
Dr. Biondi has nothing to disclose

Dr. Hansel has nothing to disclose

Dr. Murgia has nothing to disclose

Dr. D'Arpino has nothing to disclose

Dr. Tramontana has nothing to disclose

Dr. Stingeni has nothing to disclose

The manuscript was approved by the institutional review board

Patients consent statement: All patients and controls give their consent to the skin test and to the data publication

The article has not been previously published and is not currently submitted elsewhere

To the Editor,

Vaccination is crucial to contrast the spreading of the severe acute respiratory syndrome coronavirus 2 (SARS-CoV2) and the second dose is necessary to prevent the Coronavirus disease-19 (COVID-19) induced by the emerging B.1.617.2 (delta) variant. ${ }^{1}$

During clinical approval studies and post-marketing phases, mucous-cutaneous adverse reactions have been rarely observed, both local (injection site) and systemic reactions. Among the hypersensitivity reactions, immediate (anaphylaxis, urticaria-angioedema syndrome) were more frequently observed than delayed (maculopapular eruptions) reactions; moreover, anaphylaxis is a rare event, estimated at 7.9 per million doses globally. $^{2}$ The causes of these allergic reactions remain unknown, but excipients with known sensitizing potential may play a role. Particularly, Pfizer-BioNTech vaccine contains polyethylene glycol (PEG)-2000, Moderna vaccine PEG-2000 and tromethamine, AstraZeneca and Janssen vaccines polysorbate 80. PEGs and polysorbate 80 are cross-reactive and may cause mostly IgE-mediated allergic reactions, while tromethamine is responsible for both immediate and delayed reactions. ${ }^{3}$

The European Academy of Allergy and Clinical Immunology (EAACI) ${ }^{3}$ and the Italian Society of Dermatology (SIDeMaST) ${ }^{4}$ recommended, in patients with suspected allergic reaction after the first dose of SARS-CoV2 vaccine, an accurate allergologic workup, performing skin tests with vaccines and their components, in order to investigate the allergy and eventually to find potential alternative SARS-CoV2 vaccines. According to these recommendations, and considering the limits of skin test with Pfizer-BioNTech vaccine, ${ }^{5}$ we developed a skin test protocol with SARS-CoV2 vaccine excipients (Table 1). Readings were performed at $48 \mathrm{~h}$ and $96 \mathrm{~h}$ for patch test (PT), 20 min for skin prick test (SPT), and 20 min and $24 \mathrm{~h}$ for intradermal test (ID).

21 patients who referred adverse reactions to SARS-CoV2 vaccine first dose, summarized in Table 2, underwent to skin tests as previously described. None developed hypersensitivity or irritant reactions. The same tests were also performed, with negative results, in ten volunteers who had received the first dose of SARS-CoV2 vaccine and in ten volunteers who did not receive vaccine. All patients and controls referred transient local burning sensation after tromethamine ID, without skin lesions. All 21 patients then received the second dose of vaccine without relapses.

Skin tests with SARS-CoV2 vaccine excipients are not still standardized. Wolfson et al ${ }^{6}$ tested 80 patients with immediate and delayed reactions to COVID-19 vaccines using MiraLAX and methylprednisolone acetate (both containing PEG-3350) for SPT and ID, and triamcinolone acetonide or Refresh Tears (both containing polysorbate 80 ) for SPT and ID. They observed 14 (17.5\%) positive reactions, 12 of them with Refresh Tears, which was found to be an irritant in $52 \%$ of nonallergic controls. $75 \%$ of patients with negative skin test had the second vaccine dose without recurrent symptoms, while among the 14 patients with positive skin tests, 10 received the second dose, 3 of whom with recurrence of allergic reactions and 7 without relapses. 
The Authors concluded that excipient skin testing "not impact tolerance of a second dose in patients with immediate or delayed reactions". Differently, we tested the pure excipients since the use of drugs can increase the risk of irritant and false positive reactions due to the presence of other components, including the active pharmaceutical ingredient. Moreover, the use of corticosteroids may lead to false negative reactions, due to their immunosuppressant effect. Finally, we tested PEG-2000 and not PEG-3350, since the molecular weight could influence the immunoreactivity of the compound.

A recent consensus ${ }^{2}$ suggests against performing skin testing using SARS-CoV2 vaccines or excipients in patients with a history of anaphylaxis to SARS-CoV2 vaccine, because of their unknown sensitivity/specificity in predicting severe allergic reactions. As a matter of fact, our skin test protocol, conducted with pure excipients, demonstrated good tolerability and seems to be useful in patients who reported mucous-cutaneous adverse reactions after the first dose of SARS-CoV2 vaccine, despite the negative predictive value must be confirmed in larger cohort of patients.

TABLE 1. Skin test

\begin{tabular}{llll}
\hline Excipient & Patch test & Skin prick test & Intradermal test \\
\hline Polyethylene glycol 2000 & $5 \%$ pet & $0.01 \%, 0.1 \%, 1 \%$ et & $0.01 \% \mathrm{aq}$ \\
Polysorbate 80 & $5 \%$ pet & $0.01 \%, 0.1 \%, 1 \%$ et & $0.01 \% \mathrm{aq}$ \\
Tromethamine & $1 \% \mathrm{aq}$ & $0.1 \%$ et & $0.01 \% \mathrm{aq}$ \\
\hline
\end{tabular}

aq: in saline; et: in ethanol; pet: in petrolatum

\begin{tabular}{|c|c|c|c|c|c|c|c|}
\hline Patient $\mathbf{n}^{\circ}$ & $\begin{array}{l}\text { Gender, } \\
\text { age }\end{array}$ & $\begin{array}{l}\text { Personal } \\
\text { atopic } \\
\text { history }\end{array}$ & $\begin{array}{l}\text { Allergy } \\
\text { history }\end{array}$ & $\begin{array}{l}\text { Anti } \\
\text { SARS- } \\
\text { CoV-2 } \\
\text { vaccine }\end{array}$ & $\begin{array}{l}\text { Type of } \\
\text { reaction }\end{array}$ & $\begin{array}{l}\text { Time of } \\
\text { onset }\end{array}$ & Treatment \\
\hline 1 & $\mathrm{~F}, 24$ & $\begin{array}{l}\text { Allergic } \\
\text { rhinitis }\end{array}$ & None & $\begin{array}{l}\text { Pfizer- } \\
\text { BioNTech }\end{array}$ & $\begin{array}{l}\text { Acute } \\
\text { urticaria }\end{array}$ & 5 minutes & $\begin{array}{l}\text { Systemic } \\
\text { antihistamine }\end{array}$ \\
\hline 2 & $\mathrm{~F}, 31$ & $\begin{array}{l}\text { Allergic } \\
\text { rhinitis }\end{array}$ & None & $\begin{array}{l}\text { Pfizer- } \\
\text { BioNTech }\end{array}$ & $\begin{array}{l}\text { Angioedema } \\
\text { (tongue, } \\
\text { gums) }\end{array}$ & 24 hours & None \\
\hline 3 & M, 28 & $\begin{array}{l}\text { Allergic } \\
\text { rhinitis }\end{array}$ & None & $\begin{array}{l}\text { Pfizer- } \\
\text { BioNTech }\end{array}$ & $\begin{array}{l}\text { Acute } \\
\text { urticaria }\end{array}$ & 5 minutes & None \\
\hline 4 & $\mathrm{~F}, 58$ & $\begin{array}{l}\text { Allergic } \\
\text { rhinitis } \\
\text { Asthma }\end{array}$ & None & $\begin{array}{l}\text { Pfizer- } \\
\text { BioNTech }\end{array}$ & $\begin{array}{l}\text { Face } \\
\text { erythema }\end{array}$ & $\begin{array}{l}30 \\
\text { minutes }\end{array}$ & None \\
\hline 5 & $\mathrm{~F}, 50$ & $\begin{array}{l}\text { Allergic } \\
\text { rhinitis }\end{array}$ & None & $\begin{array}{l}\text { Pfizer- } \\
\text { BioNTech }\end{array}$ & $\begin{array}{l}\text { Face } \\
\text { erythema }\end{array}$ & $\begin{array}{l}20 \\
\text { minutes }\end{array}$ & None \\
\hline 6 & $\mathrm{~F}, 44$ & $\begin{array}{l}\text { Allergic } \\
\text { rhinitis } \\
\text { Atopic } \\
\text { dermatitis }\end{array}$ & $\begin{array}{l}\text { Contact } \\
\text { allergy } \\
\text { (nichel } \\
\text { sulphate, } \\
\text { fragrances) }\end{array}$ & $\begin{array}{l}\text { Pfizer- } \\
\text { BioNTech }\end{array}$ & $\begin{array}{l}\text { Angioedema } \\
\text { (tongue, } \\
\text { lips) }\end{array}$ & 10 minutes & None \\
\hline 7 & $\mathrm{~F}, 49$ & $\begin{array}{l}\text { Allergic } \\
\text { rhinitis }\end{array}$ & $\begin{array}{l}\text { Contact } \\
\text { allergy } \\
\text { (nichel } \\
\text { sulphate) }\end{array}$ & $\begin{array}{l}\text { Pfizer- } \\
\text { BioNTech }\end{array}$ & $\begin{array}{l}\text { Anaphylactoid } \\
\text { reaction }\end{array}$ & 20 minutes & $\begin{array}{l}\text { Systemic an- } \\
\text { tihistamines } \\
\text { and } \\
\text { corticosteroid }\end{array}$ \\
\hline
\end{tabular}




\begin{tabular}{|c|c|c|c|c|c|c|c|}
\hline Patient $\mathbf{n}^{\circ}$ & $\begin{array}{l}\text { Gender, } \\
\text { age }\end{array}$ & $\begin{array}{l}\text { Personal } \\
\text { atopic } \\
\text { history }\end{array}$ & $\begin{array}{l}\text { Allergy } \\
\text { history }\end{array}$ & $\begin{array}{l}\text { Anti } \\
\text { SARS- } \\
\text { CoV-2 } \\
\text { vaccine }\end{array}$ & $\begin{array}{l}\text { Type of } \\
\text { reaction }\end{array}$ & $\begin{array}{l}\text { Time of } \\
\text { onset }\end{array}$ & Treatment \\
\hline 8 & $\mathrm{~F}, 32$ & $\begin{array}{l}\text { Allergic } \\
\text { rhinitis } \\
\text { Asthma }\end{array}$ & $\begin{array}{l}\text { Food allergy } \\
\text { (milk and } \\
\text { fish } \\
\text { proteins) }\end{array}$ & $\begin{array}{l}\text { Pfizer- } \\
\text { BioNTech }\end{array}$ & $\begin{array}{l}\text { Asthma } \\
\text { exacerbation }\end{array}$ & 13 hours & $\begin{array}{l}\text { Inalatory } \\
\text { formoterol } \\
\text { and } \\
\text { beclomethasor }\end{array}$ \\
\hline 9 & $\mathrm{~F}, 39$ & $\begin{array}{l}\text { Allergic } \\
\text { rhinitis }\end{array}$ & $\begin{array}{l}\text { Contact } \\
\text { allergy } \\
\text { (nichel } \\
\text { sulphate) }\end{array}$ & $\begin{array}{l}\text { Pfizer- } \\
\text { BioNTech }\end{array}$ & $\begin{array}{l}\text { Reflex } \\
\text { syncope }\end{array}$ & 20 minutes & $\begin{array}{l}\text { Intravenous } \\
\text { saline } \\
\text { solution, } \\
\text { oxygen }\end{array}$ \\
\hline 10 & $\mathrm{~F}, 50$ & $\begin{array}{l}\text { Allergic } \\
\text { rhinitis }\end{array}$ & $\begin{array}{l}\text { Urticaria- } \\
\text { angioedema } \\
\text { (penicillin) }\end{array}$ & $\begin{array}{l}\text { Pfizer- } \\
\text { BioNTech }\end{array}$ & $\begin{array}{l}\text { Reflex } \\
\text { syncope }\end{array}$ & 2-3 minutes & $\begin{array}{l}\text { Systemic an- } \\
\text { tihistamines } \\
\text { and } \\
\text { corticosteroids }\end{array}$ \\
\hline 11 & $\mathrm{~F}, 72$ & $\begin{array}{l}\text { Allergic } \\
\text { rhinitis } \\
\text { Asthma }\end{array}$ & $\begin{array}{l}\text { Contact } \\
\text { allergy } \\
\text { (nichel } \\
\text { sulphate, } \\
\text { potassium } \\
\text { bichromate) }\end{array}$ & $\begin{array}{l}\text { Pfizer- } \\
\text { BioNTech }\end{array}$ & $\begin{array}{l}\text { Angioedema } \\
\text { (eyelids) }\end{array}$ & 1 hour & $\begin{array}{l}\text { Systemic an- } \\
\text { tihistamines } \\
\text { and } \\
\text { corticosteroids }\end{array}$ \\
\hline 12 & $\mathrm{~F}, 75$ & None & $\begin{array}{l}\text { Contact } \\
\text { allergy } \\
\text { (nichel } \\
\text { sulphate) } \\
\text { Food allergy } \\
\text { (tomato } \\
\text { proteins) }\end{array}$ & $\begin{array}{l}\text { Pfizer- } \\
\text { BioNTech }\end{array}$ & $\begin{array}{l}\text { Angioedema } \\
\text { (face) }\end{array}$ & 72 hours & $\begin{array}{l}\text { Systemic } \\
\text { antihistamines }\end{array}$ \\
\hline 13 & $\mathrm{~F}, 49$ & $\begin{array}{l}\text { Allergic } \\
\text { rhinitis }\end{array}$ & $\begin{array}{l}\text { Contact } \\
\text { allergy } \\
\text { (nichel } \\
\text { sulphate) }\end{array}$ & $\begin{array}{l}\text { Pfizer- } \\
\text { BioNTech }\end{array}$ & $\begin{array}{l}\text { Face } \\
\text { erythema }\end{array}$ & 2-3 minutes & $\begin{array}{l}\text { Systemic an- } \\
\text { tihistamines } \\
\text { and } \\
\text { corticosteroids }\end{array}$ \\
\hline 14 & $\mathrm{~F}, 59$ & $\begin{array}{l}\text { Allergic } \\
\text { rhinitis }\end{array}$ & None & $\begin{array}{l}\text { Pfizer- } \\
\text { BioNTech }\end{array}$ & $\begin{array}{l}\text { Anaphylactoid } \\
\text { reaction }\end{array}$ & $\begin{array}{l}30 \\
\text { minutes }\end{array}$ & $\begin{array}{l}\text { Systemic } \\
\text { antihis- } \\
\text { tamines } \\
\text { and } \\
\text { corticosteroids }\end{array}$ \\
\hline 15 & $\mathrm{~F}, 53$ & $\begin{array}{l}\text { Allergic } \\
\text { rhinitis }\end{array}$ & None & $\begin{array}{l}\text { Pfizer- } \\
\text { BioNTech }\end{array}$ & $\begin{array}{l}\text { Angioedema } \\
\text { (tongue) }\end{array}$ & 2-3 minutes & $\begin{array}{l}\text { Systemic } \\
\text { antihistamines }\end{array}$ \\
\hline 16 & $\mathrm{~F}, 54$ & $\begin{array}{l}\text { Allergic } \\
\text { rhinitis }\end{array}$ & None & $\begin{array}{l}\text { Pfizer- } \\
\text { BioNTech }\end{array}$ & $\begin{array}{l}\text { Urticaria- } \\
\text { angioedema, } \\
\text { bullous } \\
\text { injection site } \\
\text { reaction }\end{array}$ & 12 hours & $\begin{array}{l}\text { Systemic an- } \\
\text { tihistamines } \\
\text { and } \\
\text { corticosteroids }\end{array}$ \\
\hline 17 & $\mathrm{~F}, 31$ & None & None & AstraZeneca & $\begin{array}{l}\text { Maculopapular } \\
\text { rash }\end{array}$ & 72 hours & None \\
\hline 18 & $\mathrm{~F}, 74$ & None & None & AstraZeneca & $\begin{array}{l}\text { Maculopapular } \\
\text { rash }\end{array}$ & 24 hours & $\begin{array}{l}\text { Systemic } \\
\text { corticosteroids }\end{array}$ \\
\hline
\end{tabular}




\begin{tabular}{|c|c|c|c|c|c|c|c|}
\hline Patient $\mathbf{n}^{\circ}$ & $\begin{array}{l}\text { Gender, } \\
\text { age }\end{array}$ & $\begin{array}{l}\text { Personal } \\
\text { atopic } \\
\text { history }\end{array}$ & $\begin{array}{l}\text { Allergy } \\
\text { history }\end{array}$ & $\begin{array}{l}\text { Anti } \\
\text { SARS- } \\
\text { CoV-2 } \\
\text { vaccine }\end{array}$ & $\begin{array}{l}\text { Type of } \\
\text { reaction }\end{array}$ & $\begin{array}{l}\text { Time of } \\
\text { onset }\end{array}$ & Treatment \\
\hline 19 & $\mathrm{~F}, 45$ & $\begin{array}{l}\text { Allergic } \\
\text { rhinitis }\end{array}$ & $\begin{array}{l}\text { Contact } \\
\text { allergy } \\
\text { (nichel } \\
\text { sulphate) }\end{array}$ & AstraZeneca & $\begin{array}{l}\text { Urticaria- } \\
\text { angioedema }\end{array}$ & 10 hours & $\begin{array}{l}\text { Systemic an- } \\
\text { tihistamines } \\
\text { and } \\
\text { corticosteroids }\end{array}$ \\
\hline 20 & $\mathrm{M}, 24$ & $\begin{array}{l}\text { Allergic } \\
\text { rhinitis } \\
\text { Asthma }\end{array}$ & None & Moderna & $\begin{array}{l}\text { Asthma ex- } \\
\text { acerbation, } \\
\text { acute } \\
\text { urticaria }\end{array}$ & 20 minutes & $\begin{array}{l}\text { Systemic } \\
\text { corticosteroids }\end{array}$ \\
\hline 21 & $\mathrm{~F}, 35$ & None & $\begin{array}{l}\text { Contact } \\
\text { allergy } \\
\text { (parapheny }\end{array}$ & $\begin{array}{l}\text { Moderna } \\
\text { diamine) }\end{array}$ & $\begin{array}{l}\text { Reflex } \\
\text { syncope }\end{array}$ & 2-3 minutes & None \\
\hline
\end{tabular}

\section{References}

1. Lopez Bernal J, Andrews N, Gower C, et al. Effectiveness of Covid-19 Vaccines against the B.1.617.2 (Delta) Variant. N Engl J Med . 2021;385(7):585-594.

2. Greenhawt M, Abrams EM, Shaker M, et al. The Risk of Allergic Reaction to SARS-CoV-2 Vaccines and Recommended Evaluation and Management: A Systematic Review, Meta-Analysis, GRADE Assessment, and International Consensus Approach. J Allergy Clin Immunol Pract . 2021;18:S22132198(21)00671-1.

3. Sokolowska M, Eiwegger T, Ollert M, et al. EAACI statement on the diagnosis, management and prevention of severe allergic reactions to COVID-19 vaccines. Allergy . 2021;76(6):1629-1639.

4. Stingeni L, Bianchi L, Zalaudek I, et al. Adverse cutaneous and mucous reactions from anti SARS-CoV2 vaccines: recommendations from the Italian Society of Dermatology (SIDeMaST). Ital J Dermatol Venerol . 2021;156(2):115-117.

5. Bianchi L, Biondi F, Hansel K, et al. Skin tests in urticaria/angioedema and flushing to PfizerBioNTech SARS-CoV-2 vaccine: Limits of intradermal testing. Allergy . 2021;76(8):2605-2607.

6. Wolfson AR, Robinson LB, Li L, McMahon AE, Cogan AS, Fu X, et al. First-Dose mRNA COVID-19 Vaccine Allergic Reactions: Limited Role for Excipient Skin Testing. J Allergy Clin Immunol Pract . 2021:S2213-2198(21)00675-9. 